\title{
VALIDACIÓN DE LA ESCALA DE VULNERABILIDAD HACIA LOS MIGRANTES EN POBLACIÓN DEL NOROESTE DE MÉXICO
}

\section{VALIDATION OF THE SCALE OF VULNERABILITY TO MIGRANTS IN THE POPULATION OF NORTHWEST MEXICO}

Fernanda Guadalupe Rascón Arriaga*, Nissa Yaing Torres Soto**, Héctor Francisco Vega Deloya.*

Universidad de Sonora*, Universidad de Quintana Roo**

Correspondencia:fergpe_93@hotmail.com

\section{RESUMEN}

El desplazo de migrantes extranjeros por México ha incrementado, y a su vez, el aumento y reproducción de la vulnerabilidad estructural y cultural hacia los migrantes. El objetivo de la investigación fue diseñar y validar la escala de vulnerabilidad hacia los migrantes como sujeto de derecho. Se realizó un estudio cuantitativo, no experimental, transversal, descriptivo y correlacional con una muestra de 233 participantes de los cuales 142 (60.94\%) fueron mujeres y 91 (39.05\%) fueron hombres con una edad promedio de 29.05 ( $\mathrm{DE}=10.70$ ) de una ciudad del noroeste de México. Los resultados del análisis factorial exploratorio reveló la existencia de dos dominios para la escala de vulnerabilidad hacia el migrante (vulnerabilidad estructural y vulnerabilidad cultural) que explicaron el $51.90 \%$ de la varianza total. Un análisis de covarianzas de dos factores y un modelo de segundo orden produjeron índices de bondad de ajuste adecuados que evidenciaron validez convergente de constructo. La escala total produjo un índice de consistencia interna elevado $(\alpha=.93)$ al igual que las subescalas. La correlación significativa entre estos factores arrojó evidencias 
de validez concurrente de la escala. Finalmente, el análisis de varianzas mostró diferencias estadísticamente significativas en la percepción hacia la vulnerabilidad estructural y cultural por ingreso mensual familiar.

Palabras clave: vulnerabilidad, migrantes, derechos humanos.

\section{ABSTRACT}

The displacement of foreign migrants through Mexico has increased, and in turn, the increase and reproduction of structural and cultural vulnerability towards migrants. The objective of this research was to design and validate the scale of vulnerability towards migrants as a subject of law. A quantitative, non-experimental, cross-sectional, descriptive and correlational study was carried out with a sample of 233 participants of northwest of Mexico with 142 (60.94\%) women and 91 (39.05\%) men with an average age of $29.05(\mathrm{SD}=10.70)$. The results of the exploratory factor analysis revealed existence of two domains for the scale of vulnerability towards the migrant (structural vulnerability and cultural vulnerability) that explained 51.90\% of the total variance. A two-factor analysis of covariances and a secondorder model produced adequate goodness-of-fit indices that evidenced convergent construct validity. The total scale produced a high internal consistency index $(\alpha=.93)$ as did the subscales. The significant correlation between these factors yielded evidence of concurrent validity of the scale. Finally, the analysis of variances showed statistically significant differences in the perception of structural and cultural vulnerability due to monthly family income.

Key words: vulnerability, migrants, human rights.

\section{INTRODUCCIÓN}

El concepto de vulnerabilidad es utilizado en disciplinas como la geografía, agronomía, sociología, psicología y geofísica (Adger, 2006; Brooks, 
2003; Cutter, 1996; Kasperson y Dow, 2005; París, 2012). El concepto de vulnerabilidad lo define Aday (1994) como el riesgo físico, psicológico $\mathrm{y}$ de salud social al que suelen someterse las personas en condiciones de desventaja en un determinado contexto sociocultural. La población más vulnerable generalmente son las madres, los infantes, los enfermos crónicos y discapacitados, los sujetos con VIH/SIDA, los enfermos mentales; las personas que consumen algún tipo de sustancia psicoactiva, los individuos propensos al suicidio $\mathrm{u}$ homicidio; los pertenecientes a familias abusadoras, los vagabundos y entre ellos, los migrantes y refugiados (Aday, 1994). Por lo tanto, la vulnerabilidad de los migrantes representa un grupo poblacional vulnerable con carencia de protección social.

Dentro del campo migratorio, la vulnerabilidad se entiende como la exposición diferencial a un conjunto de riesgos sociales y ambientales, así como la capacidad de adaptación que desarrollan los migrantes para hacer frente a determinados riesgos como el asalto, deshidratación al cruzar al desierto, así como violaciones a los derechos humanos por los servidores públicos (Paris, 2012). Esto se debe a que la estructura gubernamental no desarrolla y aplica las políticas necesarias para proteger a los migrantes con base a sus derechos humanos (ONU, 2003).

La vulnerabilidad proviene de situaciones disposicionales y contextuales. Por un lado, los migrantes de distinto origen y raza, se enfrenta a un recorrido migratorio que cambia sus perspectivas de vida, son susceptibles a padecer enfermedades crónicas y mentales relacionadas con el estrés y, por consiguiente, sus costumbres y tradiciones. Por otro lado, los migrantes deciden mejorar su situación de vida. Durante el tránsito y el destino, los mexicanos y centroamericanos mantienen la expectativa de mejorar sus condiciones de vida, pero a su vez, mantienen la incertidumbre que durante 
su trayectoria son susceptibles a presentar carencias de carácter económico y social (Hernández-Rosete, Sanchez, Pelcastre, y Juárez, 2005).

La vulnerabilidad hacia el migrante como sujeto derecho, según Bustamante (2003, p. 31) refiere a "la condición social de ausencia de poder, concomitante a la impunidad de los que violan los derechos humanos". El concepto presenta una dimensión estructural, entendiéndose como "la diferencia entre un nacional y un extranjero/inmigrante en sus relaciones con el Estado en el país de acogida" (Bustamante, 2010, p. 226); por la carencia extrema del poder que se fundamenta desde un punto de vista objetivo. Y una dimensión cultural de carácter subjetiva que se define como "el conjunto de elementos culturales (estereotipos, prejuicios, racismo, xenofobia, ignorancia y discriminación institucional) con significados despectivos que tienden a justificar las diferencias de poder entre los nacionales y los extranjeros o inmigrantes" (Bustamante, 2010, p. 236) y el ejercicio de la vulnerabilidad (Bustamante, 2003). Comprende aspectos sociales, biológicos y condiciones estructurales del contexto (Salgado, González, Bojorquez, y Infante, 2007).

Por su parte, Quiroz (2014) señala que la vulnerabilidad del migrante se sostiene e incrementa por el reforzamiento del control migratorio y la falta de sanciones por parte del gobierno ante la violación de los derechos humanos de los migrantes. Casillas (2011) considera que la vulnerabilidad se debe a la impunidad que tiene el agresor. Además de la vulnerabilidad estructural que brinda el Estado por sus normas, existe la vulnerabilidad cultural alimentada por los valores, ideas, prejuicios e ideologías que se tienen sobre los migrantes, que justifica la diferenciación entre un nacional y un extranjero dejando a la vista comportamientos o actos desiguales entre nacionales y extranjeros (Quiroz, 2014; Pizarro, 2008). Mantener un 
trato desigual propicia que los migrantes tengan diferencia en el acceso de los bienes y servicios que brinda el Estado (Quiroz, 2014). De modo que la percepción que tenga la población nacional del migrante guia el comportamiento que se ejerce hacia el extranjero.

Además resulta importante señalar que la vulnerabilidad del migrantes se debe considerar la forma de ser y vivir según el rol social y trayectoria personal de vida. El grado de vulnerabilidad se presenta conforme las condiciones históricas y sociales del país de origen del migrante extranjero, sumado a las características individuales como la edad, etnia, sexo, escolaridad, normas sociales, motivos del desplazamiento (Quiroz, 2013), género y experiencia del viaje (Casillas, 2011).

Existen estudios que ha puesto en evidencia la vulnerabilidad del migrante, por ejemplo, Hernández-Rosete, et al. (2005) señala que los migrantes extranjeros clandestinos son vulnerables con respecto a su salud, ya que son más proclives a contraer el Virus de Inmunodeficiencia Humana (VIH), por la restricción de las políticas entre fronteras que promueven la violación de los derechos humanos a través del trabajo sexual, el tráfico de personas y la violencia en general. Por otro lado, Casillas, (2011) menciona que la red ferroviaria ha sido un espacio de negociación y complicidad con el crimen organizado para el secuestro, la trata y el desprecio de personas que ponen en riesgo la vida del migrante.

Adicionalmente, Paris (2012) indica que este colectivo generalmente es susceptible a presentar agresiones de carácter físico y verbal cuando son detenidos en el cruce fronterizo a los Estados Unidos, siendo los hombres entre 20 y 24 años, principalmente, las personas más vulnerables de acuerdo a las condiciones del cruce fronterizo, la acción social y las relaciones de 
poder que se gestan durante el trayecto, las políticas migratorias y control fronterizo.

A partir de lo anterior, se considera pertinente crear un instrumento que permita medir la percepción de los mexicanos hacia los migrantes extranjeros. A pesar de que se han desarrollado diversas escalas que miden la vulnerabilidad en diferentes poblaciones, por ejemplo, la vulnerabilidad psicológica orientada hacia sí mismos (Sinclair y Wallston, 1999), en población infantil (Forsyth, Horwitz, Leventhal, Bruger, y Leaf, 1996; Seward, Bayliss, y Ohan, 2018) en adultos mayores (Pinsker, McFarland, y Stone, 2011), en personas con discapacidad (Sofronoff, Dark, y Stone, 2011) y en estudiantes (Nogueira, Barros, y Sequeira, 2017), este concepto ha sido mayormente fundamentado desde los estudios cualitativos (Casillas, 2011; González, 2010; Hernández-Rosete et al., 2005; Quiroz, 2014; Pineda, 2020); por lo tanto, se desarrolló la escala de vulnerabilidad hacia los migrantes, a través de los componentes de vulnerabilidad estructural y vulnerabilidad cultural que parten de la propuesta teórica de Bustamante (2010).

De acuerdo con Paris (2012), el concepto de vulnerabilidad de los migrantes está en proceso de construcción y resulta pertinente crear un instrumento que permita medirlo de manera práctica. En este sentido, el presente estudio hace una propuesta de los indicadores para medir la vulnerabilidad tomando como base la teoría de Bustamante (2010) como resultado, se obtuvo una escala con dos factores, vulnerabilidad estructural y vulnerabilidad cultural, donde se analizaron las propiedades psicométricas de la escala de vulnerabilidad hacia los migrantes en población mexicana con el objetivo de medir este concepto tangiblemente que contribuya al desarrollo empírico de este variable. 
Crear escalas capaces de medir de manera refinada las actitudes de la población respecto a la vulnerabilidad que sufre el migrante, podría resultar funcional al enmascaramiento de las consecuencias de estas actitudes negativas en la actualidad. Es decir, al conocer la postura de la población mexicana hacia el migrantes puede resultar valiosa para la creación de reformas legales que contribuyan a la protección de las personas migrantes e idear políticas tendientes a revertir las situaciones de inequidad y orientarlas hacia una real integración intercultural.

\section{MÉTODO}

El diseño del presente estudio fue de carácter cuantitativo, con diseño no experimental, transversal, descriptivo y correlacional, dado que no se manipularon las variables, sino que fueron estudiadas tal cual como se expresan en su ambiente natural (Hernández, Fernández y Baptista, 2014).

\section{Participantes}

En el presente estudio participaron un total de 233 participantes de los cuales 142 (61.7\%) fueron mujeres y 91 (39.7\%) hombres con un rango de edad entre 18 a 67 años $(M=29.05, D E=10.70)$ en una ciudad del noroeste de México. La mayoría de los participantes cuentan con educación superior (25.5\%). El ingreso mensual familiar promedio fue entre 5,000 a 10,000 pesos mexicanos. Además, los encuestados reportaron haber viajado al menos 5.99 veces al interior de la República Mexicana y 5.63 al exterior (Ver Tabla 1). 
Tabla 1. Estadísticos descriptivos de las variables sociodemográficas.

\begin{tabular}{|c|c|c|}
\hline Variable & $\mathbf{F E}$ & $\%$ \\
\hline \multicolumn{3}{|l|}{ Género } \\
\hline Masculino & 91 & $38.7 \%$ \\
\hline Femenino & 144 & $61.3 \%$ \\
\hline \multicolumn{3}{|l|}{ Edad } \\
\hline $18-29$ años & 96 & $40.9 \%$ \\
\hline $30-49$ años & 55 & $23.4 \%$ \\
\hline $50-69$ aก̃os & 84 & $35.7 \%$ \\
\hline \multicolumn{3}{|c|}{ Ingreso mensual familiar } \\
\hline$\$ 0-\$ 2,500$ & 1 & $4.0 \%$ \\
\hline$\$ 2,501-\$ 5,000$ & 65 & $27.7 \%$ \\
\hline$\$ 5,001-\$ 10,000$ & 65 & $27.7 \%$ \\
\hline$\$ 10,001-\$ 20,000$ & 67 & $28.5 \%$ \\
\hline$\$ 20,001-\$ 40,000$ & 5 & $2.1 \%$ \\
\hline$>\$ 40,001$ & 32 & $13.6 \%$ \\
\hline \multicolumn{3}{|c|}{ Veces que ha viajado al interior de la República Mexicana } \\
\hline 0 & 3 & $1.3 \%$ \\
\hline $1-3$ & 75 & $32.0 \%$ \\
\hline $4-7$ & 50 & $21.2 \%$ \\
\hline $8-10$ & 12 & $4.39 \%$ \\
\hline$>10$ & 95 & $40.4 \%$ \\
\hline \multicolumn{3}{|c|}{ Veces que ha viajado al exterior de la República Mexicana } \\
\hline 0 & 5 & $2.1 \%$ \\
\hline $1-3$ & 100 & $42.6 \%$ \\
\hline $4-7$ & 28 & $11.9 \%$ \\
\hline 8-10 & 7 & $3.0 \%$ \\
\hline$>10$ & 95 & $40.4 \%$ \\
\hline
\end{tabular}




\section{Instrumento}

A partir de la propuesta teórica de Bustamante (2010), se diseñó la escala de la vulnerabilidad de los migrantes, compuesta por la sub-escala de vulnerabilidad estructural constituida por 12 ítems y la sub-escala de vulnerabilidad cultural integrada por 28 ítems. En total la escala se conformó por 40 reactivos con una escala de respuesta de tipo Likert de cinco puntos que van de 1 (totalmente en desacuerdo) a 5 (totalmente de acuerdo).

\section{Procedimiento}

Se condujo una investigación apegada a las leyes federales, estatales y normas éticas profesionales para regular de manera competente de la planeación y ejecución del estudio, así como respetar la dignidad y bienestar de los participantes siguiendo los estándares éticos que indica la Asociación Americana de Psicología (APA, 2010) el código ético del psicólogo y la declaración de Helsinki de 1964. Previo a la aplicación del cuestionario, se solicitó el consentimiento informado de los participantes y se aclaró que los datos personales eran totalmente confidenciales para fines académicos y de investigación. Los datos fueron recolectados por medio del software Qualtrics con una duración aproximada de 30 minutos.

\section{Análisis de datos}

Se procedió a obtener la validez de los instrumentos a traves del Análisis Factorial Exploratorio (AFE) por componentes principales y rotación Varimax, donde sólo se conservaron los ítems con peso factorial $\geq .40$ (Field, 2013; Pituch y Stevens, 2016). Además, se probó el índice de la prueba de Kaiser-Meyer-Olkin (KMO), entre mayor se aproxime al valor de 1, más compactas resultan las correlaciones y se producen resultados más confiables. A su vez, se probó la significancia $(\mathrm{p}<.05)$ a través de la 
prueba de esfericidad de Barlett (Field, 2013). El número de factores fue evaluado a través del índice de varianza explicada para cada factor y de la escala total de vulnerabilidad hacia el migrante.

Con el apoyo del programa SPSS versión 25, se realizaron los análisis univariados de cada sub-escala, incluyendo las medias y desviación estándar. También se estimó el índice de consistencia interna a través del alfa de Cronbach, considerando un coeficiente mayor a .60 como confiable (Corral, Frías y González, 2001). Se realizó un análisis de correlación de Pearson con los factores resultantes de la escala, para comprobar el grado de relación entre las variables del estudio (Restrepo y González, 2007), tomando en consideración la proximidad al +1 y el índice de significancia entre 05 y .01 (González, 2009) y un análisis de varianzas (ANOVA de una vía) con prueba Tukey (Field, 2013) para identificar diferencias por el ingreso mensual familiar de los participantes.

Adicionalmente, se efectuó un Análisis Factorial Confirmatorio (AFC) para probar la validez de la escala, considerando tres tipos de indicadores (estadístico, práctico y poblacional) con la ayuda del programa EQS versión 6.0 (Bentler, 2007). Para el indicador estadístico se consideró la prueba chi cuadrada $\left(\chi^{2}\right)$, si el valor resulta $p>0,05$ se considera que el modelo presenta una bondad de ajuste estadística adecuada. Sin embargo, esta prueba es susceptible a muestras grandes, para resolver esto, se calcula la $\chi 2$ relativa que se obtiene dividiendo el índice de ajuste $\chi 2$ por los grados de libertad (Schumacker y Lomax, 2004). Considerado que los indicadores estadísticos suelen ser sensibles a muestras grandes, también se estimaron los indicadores prácticos tales como el Índice de Ajuste Comparativo (CFI), Bentler-Bonett de Ajuste Normado (BBNFI) y No-Normado (BBNNFI) con valores iguales o mayores a 90 (Bentler y Bonet, 1980; Corral et al., 
2001), y por último, se estimó el índice de ajuste poblacional denominado Error de Aproximación Cuadrático Medio (RMSEA) considerando como aceptable un valor igual o menor a .06 (Hu y Bentler, 1999). Se realizaron dos modelos estructurales, el primero representó la covarianza entre los dos factor de primer orden (vulnerabilidad estructural y vulnerabilidad cultural) los cuales emergieron de forma coherente a partir de sus indicadores manifiestos, donde la covarianza entre los factores resultó alta y significativa. El segundo modelo representó los factores de primer orden denominados "vulnerabilidad estructural" y "vulnerabilidad cultural" con sus correspondientes parcelas (ítems), que conformaron al factor de segundo orden denominado "vulnerabilidad hacia el migrante".

\section{RESULTADOS}

En el AFE se procedió a fijar el número a dos factores con el método de componentes principales y rotación Varimax sobre los 40 reactivos de la escala total. Durante la optimización del análisis se eliminaron 12 ítems (3, $4,8,9,11,14,15,17,19,20,21,40)$ que resultaron con pesos factoriales menores a .40 , resultando un total de 28 reactivos que explica el $51.90 \%$ de la varianza total, con un índice de consistencia interna aceptable de .93. Los valores de comunalidad resultaron superiores a .50 (57.14\%), lo que indica que la solución factorial logró explicar altas proporciones de varianza para cada uno de los ítems sometidos a la validación. La medida de adecuación muestral KMO resultó de 90 y la prueba de esfericidad de Bartlett de 4101.544, $p=.001$. Ambos valores muestran un ajuste adecuado del AFE.

El primer componente representa la vulnerabilidad estructural. Se integra por 7 reactivos que explican el $40.93 \%$ de la varianza con pesos factoriales que oscilan entre .44 y .72. Este factor presenta un alfa de Cronbach de 
0,68 con una media general de $M=2.68(D E=.82)$. El reactivo con la media más alta fue "Los mexicanos y los extranjeros/inmigrantes son diferentes ante la ley mexicana" $(M=3, D E=1.38)$, mientras que la media más baja fue "Si un mexicano viola los derechos humanos de los extranjeros/ inmigrantes no recibe sanción alguna" $(M=2.42, D E=1.24)$.

El segundo componente denominado, vulnerabilidad cultural, describe el $10.97 \%$ de la varianza con puntuaciones entre .58 y .83 con una consistencia interna de .96 con una media general de $M=1,92$ ( $D E=$ .79). El reactivo con el índice más alto fue "Los extranjeros/inmigrantes transportan enfermedades infectocontagiosas" $(M=2.47, D E=1.18)$. Por el contrario, los indicadores más bajos fueron "Rechazaría la presencia de un extranjero/inmigrante" ( $M=1.58, D E=.97)$ y "Las mujeres extranjeras/ inmigrantes solo pueden trabajar de niñeras" $(M=1,58, D E=0,96)$ (Ver Tabla 2).

Tabla 2. Estadísticos descriptivos, cargas factoriales y comunalidad de la escala de vulnerabilidad de los migrantes.

\begin{tabular}{|c|c|c|c|c|c|}
\hline \multicolumn{6}{|l|}{ Cargas factoriales } \\
\hline Reactivo & $M$ & $D E$ & 1 & 2 & $h^{2}$ \\
\hline $\begin{array}{l}\text { 1. México solo reconoce a los derechos humanos de } \\
\text { los mexicanos }\end{array}$ & 2.84 & 1.27 & .09 & .44 & .20 \\
\hline $\begin{array}{l}\text { 2. Los mexicanos y los extranjeros/inmigrantes son } \\
\text { diferentes ante la ley mexicana }\end{array}$ & 3.00 & 1.38 & .04 & .62 & .39 \\
\hline $\begin{array}{l}\text { 5. Cualquier persona puede violar los derechos } \\
\text { humanos de los extranjeros/inmigrantes }\end{array}$ & 2.51 & 1.43 & .11 & .49 & .25 \\
\hline $\begin{array}{l}\text { 6. Si un mexicano viola los derechos humanos de los } \\
\text { extranjeros/inmigrantes no recibe sanción alguna }\end{array}$ & 2.42 & 1.24 & .00 & .70 & .48 \\
\hline $\begin{array}{l}\text { 7. Los extranjeros/inmigrantes son invisibles ante la } \\
\text { protección México }\end{array}$ & 2.51 & 1.26 & .13 & .72 & .54 \\
\hline
\end{tabular}


$\begin{array}{lllll}2.82 & 1.36 & .03 & .66 & .43\end{array}$

de los extranjeros/inmigrantes

12. México viola los derechos humanos de los extranjeros/inmigrantes ilegales

13. Los extranjeros/inmigrantes son criminales

16. Los extranjeros/inmigrantes son secuestradores

18. Las tradiciones culturales de los extranjeros /inmigrantes son extrañas

22. Los extranjeros/inmigrantes deterioran la imagen de México

23. Los extranjeros/inmigrantes ingresan a México para cometer delitos

24. Los extranjeros/inmigrantes transportan enfermedades infectocontagiosas

25. No me gustaria tener contacto directo con los extranjeros/inmigrantes

26. Estar próxima a un extranjero/inmigrantes pone en peligro mi vida

27. Solo los mexicanos deben superarse económicamente

28. Los extranjeros/inmigrantes solo buscan la manera de estafar a los mexicanos

29. No me gustaria tener a un extranjero/inmigrante de vecino

30. Rechazaria la presencia de un extranjero /inmigrante

31. Los extranjeros/inmigrantes de origen centroamericanos son groseros

32. Los extranjeros/inmigrantes de raza negra son delincuentes

33. Los extranjeros/inmigrantes de origen centroamericano no son trabajadores $\begin{array}{lllll}1.66 & 1.01 & .80 & .17 & .67\end{array}$

$\begin{array}{lllll}2.68 & 1.25 & .18 & .67 & .48\end{array}$

$\begin{array}{lllll}1.76 & 1.01 & .70 & .20 & .53\end{array}$

$\begin{array}{lllll}1.76 & 0.95 & .69 & .18 & .51\end{array}$

$\begin{array}{lllll}2.37 & 1.15 & .58 & .11 & .35\end{array}$

$\begin{array}{lllll}2.27 & 1.08 & .62 & .12 & .40\end{array}$

$\begin{array}{lllll}2.15 & 1.04 & .72 & .05 & .53\end{array}$

$\begin{array}{lllll}2.47 & 1.18 & .69 & .06 & .49\end{array}$

$\begin{array}{lllll}1.98 & 1.13 & .79 & .09 & .64\end{array}$

$\begin{array}{lllll}1.93 & 1.12 & .80 & .15 & .66\end{array}$

$\begin{array}{lllll}1.77 & 1.14 & .63 & .07 & .40\end{array}$

$\begin{array}{lllll}1.81 & 1.02 & .82 & .08 & .69\end{array}$

$\begin{array}{lllll}1.84 & 1.12 & .79 & .12 & .64\end{array}$

$\begin{array}{lllll}1.58 & 0.97 & .83 & .17 & .73\end{array}$

$\begin{array}{lllll}2.18 & 1.05 & .79 & .02 & .63\end{array}$

$\begin{array}{lllll}1.66 & 1.01 & .80 & .17 & .67\end{array}$

$\begin{array}{lllll}1.92 & 1.03 & .73 & .00 & .53\end{array}$ 
34. Los extranjeros / inmigrantes de origen centroamericano son mentirosos

35. Los extranjeros / inmigrantes de raza negra me provocan desconfianza

36. Me dan miedo los extranjeros / inmigrantes centroamericanos

37. Las mujeres extranjeras/inmigrantes solo pueden trabajar de niñeras

38. Me gusta tener amigos que fisicamente se parecen a $\mathrm{mi}$

39.El aspecto fisico de las personas determina mi actitud hacia ellas

\begin{tabular}{lcc}
\hline Indice de consistencia interna $(\alpha)$ & .68 & .96 \\
\hline \multirow{2}{*}{ Porcentaje de varianza } & 40.9 & 10.97 \\
\hline
\end{tabular}

Factor 1= Vulnerabilidad estructural; Factor 2 = Vulnerabilidad cultural

Por otra parte, se estimó la relación entre los factores que componen la escala de vulnerabilidad hacia el migrante utilizando el análisis de correlación de Pearson. Se encontró que la vulnerabilidad estructural se correlacionó de forma moderada, positiva y significativamente con la vulnerabilidad cultural $(r=.28)$ con un nivel de significancia de $p<.01$.

\section{Diferencias de variables distributivas}

El ANOVA de una vía y mediante el estadístico de Levene se acepta la hipótesis nula de las dos sub-escalas de vulnerabilidad y se acepta que existen varianzas similares entre ellas. La variable sociodemográfica de ingreso mensual familiar representa la variable agrupadora, y la variable contraste corresponde a la vulnerabilidad estructural y cultural reflejan que existen diferencias significativas. 
Las dos sub-escalas asumieron varianzas iguales y homocedasticidad, por lo que se procedió a realizar la prueba post hoc Tukey. En el primer factor, las personas con ingreso mensual familiar entre "5 001 a 20000 pesos" $(\mathrm{M}=2.55)$ perciben menor vulnerabilidad estructural hacia el extranjero respecto al grupo de "más de 20000 pesos" $(\mathrm{M}=3.10)$. Mientras que el grupo con ingresos "de 0 a 5000 pesos" no presentó diferencias estadísticamente significativas con los grupos anteriores ( $\mathrm{p}>.05)$.

En el segundo factor, el grupo "de 0 a 5000 pesos" $(\mathrm{M}=2.10)$ presentó mayor vulnerabilidad cultural en comparación con el grupo "de 5001 a 20000 pesos" $(M=1.72)$. Pero no presentaron diferencias significativas (p>.05) con el grupo de "más de 20000 pesos". Por su parte, el grupo con ingresos entre "5 001 a 20000 pesos" $(\mathrm{M}=1.72)$ perciben menos vulnerable al migrante extranjero en contraste con el grupo de "más de 20000 pesos" $(\mathrm{M}=2.28)$. En cambio, con el grupo "de 0 a 5000 pesos" no se presentaron diferencias significativas $(\mathrm{p}>$.05) (Ver Tabla 3).

Tabla 3. Diferencias y valores de media por nivel de ingreso mensual familiar para las sub-escala de vulnerabilidad hacia los migrantes.

\section{Nivel de ingreso mensual familiar}

\begin{tabular}{|c|c|c|c|c|c|c|c|}
\hline \multirow[t]{2}{*}{ Sub-escalas } & \multicolumn{2}{|c|}{$\begin{array}{c}\text { De } 0 \text { a } 5000 \\
\text { pesos }\end{array}$} & \multicolumn{2}{|c|}{$\begin{array}{c}\text { De } 5001 \text { a } 20000 \\
\text { pesos }\end{array}$} & \multicolumn{2}{|c|}{$\begin{array}{l}\text { Más de } 20 \\
000 \text { pesos }\end{array}$} & \multirow[t]{2}{*}{ Valor $p$} \\
\hline & $M$ & $D E$ & $\boldsymbol{M}$ & $D E$ & $M$ & $D E$ & \\
\hline $\begin{array}{l}\text { Vulnerabilidad } \\
\text { estructural }\end{array}$ & $2.72_{b}$ & 0.76 & $2.55_{\mathrm{a}}$ & 0.85 & $3.10_{\mathrm{a}}$ & 0.74 & .003 \\
\hline $\begin{array}{l}\text { Vulnerabilidad } \\
\text { cultural }\end{array}$ & $2.10_{\mathrm{a}}$ & 0.91 & $1.72_{\mathrm{ab}}$ & 0.55 & $2.28 \mathrm{~b}$ & 1.02 & .001 \\
\hline
\end{tabular}

Nota: Si comparten literales iguales son estadísticamente diferentes $(p<.05)$ 


\section{Análisis factorial confirmatorio}

La Figura 1, muestra los resultados del modelo de covarianzas de la escala de vulnerabilidad hacia el migrante. Todos los indicadores observables (ítems) se cargaron significativamente $(p<.05)$ con los factores de primer orden correspondientes (vulnerabilidad estructural y vulnerabilidad cultural), lo que indica validez de constructo convergente para la escala. El factor de la vulnerabilidad estructural se constituyó por 7 reactivos, y el segundo factor, vulnerabilidad cultural, se conformó por 21, todos los indicadores presentaron cargas factoriales altas y significativas. La covarianza entre la vulnerabilidad estructural y vulnerabilidad cultural resultó significativa $(p<.05)$ con una bondad de ajuste aceptable $(\chi 2=577,691, g . l .303, p=0,0001$, $\chi 2 / g . l .=1.90 ; B B N F I=0,86 ; B B N N F I=0,92 ; C F I=0,93, R M S E A=0,06)$. Lo anterior demuestra que el modelo teórico es compatible con los datos empíricos. 


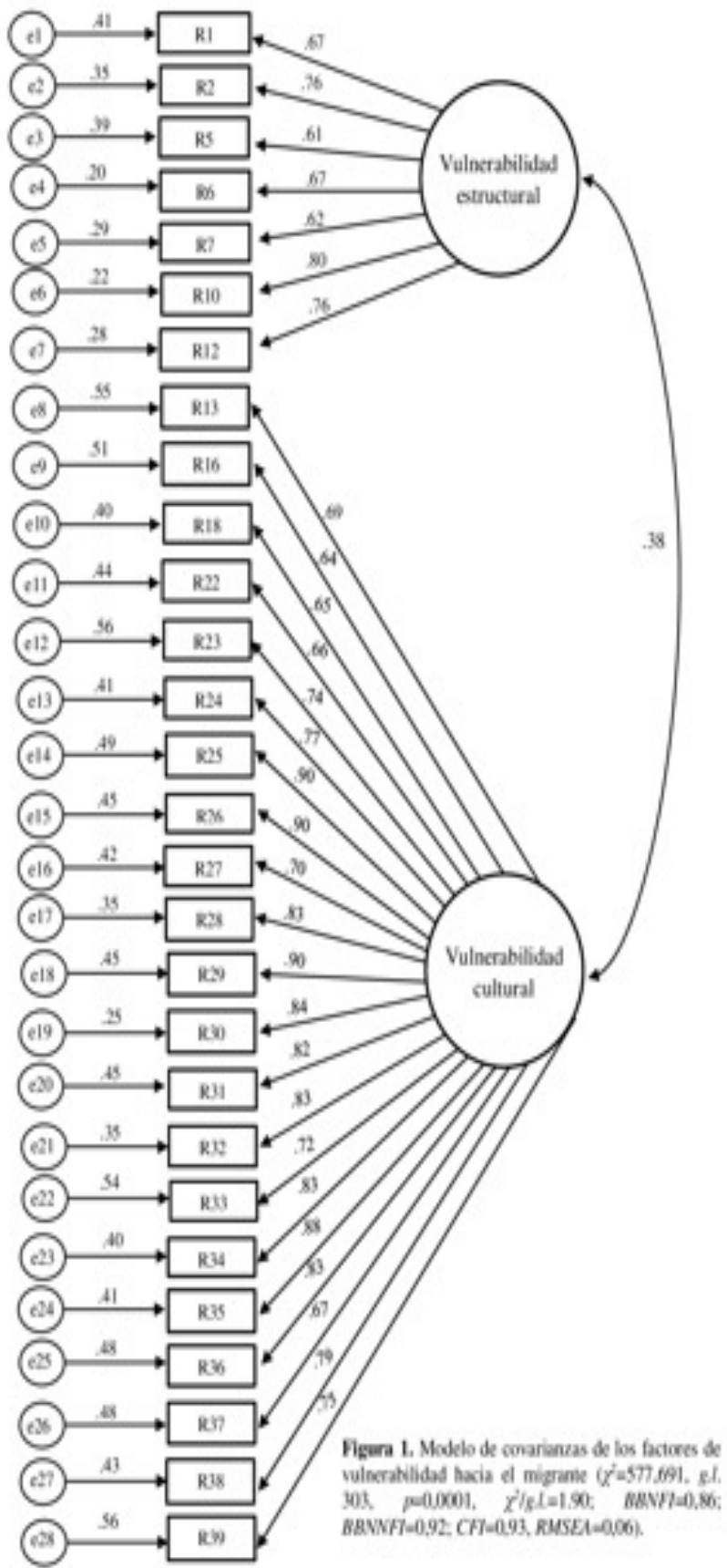


Debido a que la sub-escala de vulnerabilidad cultural se conformó por un total de 21 reactivos en comparación con la sub-escala de vulnerabilidad estructural que solo estuvo conformada por 7 ítems, se consideró oportuno realizar parcelas que conformaran grupos de ítems para someter a prueba un modelo de segundo orden. Emplear parcelas dentro de un modelo de ecuaciones estructurales posee varias ventajas, mayor confiabilidad y relación entre el número de casos por parámetro, comunalidades más altas, y menos probabilidad de violar supuestos y fuentes de error de muestreo (Little, Cunningham, Shahar y Widaman, 2002).

En la Figura 2, se probó un modelo segundo orden donde los factores de primer orden (vulnerabilidad estructural y vulnerabilidad cultural) conformaron al factor denominado "vulnerabilidad hacia los migrantes". El modelo presentó validez convergente de constructo ya que las relaciones de los factores de primero orden resultaron altas y significativas. La bondad de ajuste del modelo resultó no significativa $\left(\chi^{2}=10.54,5 \mathrm{gl} ., p=.06\right), \mathrm{y}$ los indicadores prácticos fueron aceptables $(B B N F I=.99 ; B B N N F I=.98$; $C F I=.99)$, al igual que el $R M S E A$ (.06). Lo anterior indica que el modelo teórico no es estadísticamente diferente a los datos empíricos.

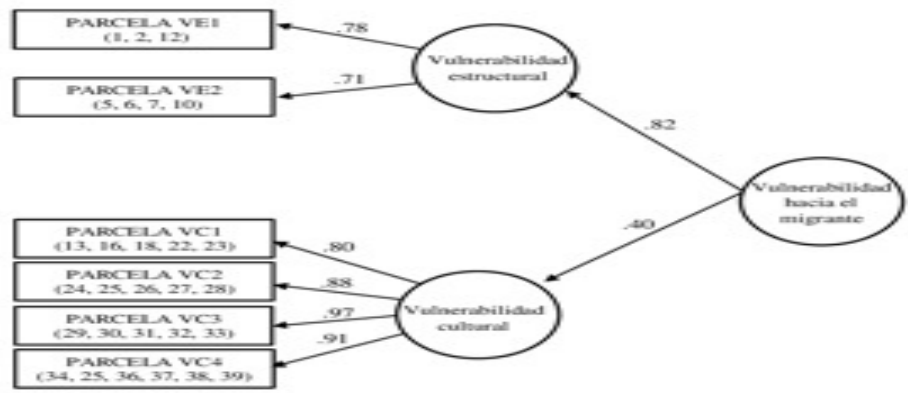

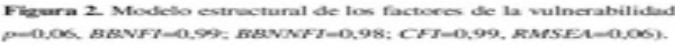


Para los índices de ajuste para modelos anidados, donde se compara el incremento de la bondad de ajuste práctica, estadística y poblacional, es posible apreciar que se consigue mejor bondad de ajuste cuando la escala es dividida en dos factores que están relacionados (vulnerabilidad estructural y vulnerabilidad cultural) respecto al modelo nulo donde todos los ítems son independientes y no se permiten factores comunes. En cuanto a los modelos con factor de segundo orden (con parcelas y sin parcelas), igualmente se obtuvo un adecuado ajuste y una estructura coherente en ambos modelos (Ver Tabla 4).

Tabla 4. Estadísticos de bondad de ajuste para modelos anidados

\begin{tabular}{|c|c|c|c|c|c|c|c|c|}
\hline \multirow[b]{2}{*}{ Modelo } & \multicolumn{8}{|c|}{ Índices de ajuste } \\
\hline & $g l$ & $\chi^{2}$ & $p$ & $\mathrm{X}^{2} / g l$ & $\begin{array}{c}B B N F \\
I\end{array}$ & $\begin{array}{c}B B N N F \\
I\end{array}$ & CFI & $R M S E A$ \\
\hline Nulo & 350 & 1070.68 & $<.05$ & 3.05 & .75 & .80 & .81 & .09 \\
\hline $\begin{array}{l}2 \text { factores } \\
\text { relacionados }\end{array}$ & 303 & 577.691 & $<.05$ & 1.65 & .86 & .92 & .93 & .06 \\
\hline $\begin{array}{l}1 \text { factor de } \\
\text { segundo orden } \\
\text { (con parcelas) }\end{array}$ & 5 & 10.55 & $>.05$ & 2.11 & .99 & .98 & .99 & .06 \\
\hline $\begin{array}{l}1 \text { factor de } \\
\text { segundo orden } \\
\text { (sin parcelas) }\end{array}$ & 309 & 530.09 & $<.05$ & 1.71 & .90 & .93 & .94 & .05 \\
\hline
\end{tabular}

$g l=$ grados de libertad, $\mathrm{X}^{2}=$ chi cuadrado, $\mathrm{p}=$ significancia, $\mathrm{X}^{2} / g l=$ chi cuadrado entre los grados de libertad, $B B N F I=$ Bentler-Bonett de Ajuste Normado, $B B N N F I=$ Bentler-Bonett de Ajuste No Normado, $C F I=$ Índice de Ajuste Comparativo, RMSEA = Error de Aproximación Cuadrático Medio.

\section{DISCUSIÓN}

En este estudio se planteó como objetivo diseñar y analizar las evidencias de validez y confiabilidad de un instrumento para evaluar la vulnerabilidad hacia el migrante a partir de la percepción que tienen las personas del 
noroeste de México. Para lograr dicho objetivo, se aplicaron diferentes pruebas estadísticas bajo el supuesto de que cada prueba cumple con un objetivo particular.

Los resultados de los análisis demuestran que el instrumento desarrollado para medir la vulnerabilidad hacia el migrante con base en la propuesta teórica de Bustamante (2010) presenta propiedades psicométricas que los hacen pertinente para medir este constructo en población general del noroeste de México. Esta obtuvo altos índices de consistencia interna adecuados tanto para la escala total $(\alpha=.93)$ y las sub-escalas de vulnerabilidad estructural $(\alpha=.68)$ y vulnerabilidad cultural $(\alpha=.96)$.

Los hallazgos confirman la pertinencia de un modelo bidimensional de medición de la vulnerabilidad hacia el migrante constituida por la vulnerabilidad estructural (dimensión objetiva) y la vulnerabilidad cultural (dimensión subjetiva) en concordancia con la propuesta teórico de Bustamante (2010). El factor de vulnerabilidad estructural se compone por 7 indicadores finales, los ítems que no presentaron un peso factorial significativo en el $\operatorname{AFE}(3,4,8,9,11)$ fueron eliminados, se considera que esto se debe a que los reactivos iban en sentido de migrantes residentes de México que contaban con un estatus legal dentro del país, por lo tanto, el sentido de ellos era contradictorio con el resto de los indicadores.

Los indicadores que conforman el factor de vulnerabilidad estructural demuestran las relaciones de poder, políticas migratorias e impunidad por parte del agresor en contra de los migrantes extranjeros, es decir, la desigualdad que caracteriza a los migrantes donde se le asigna un papel carente de poder frente a la las leyes mexicanas. Casillas (2011), Paris (2012) y Quiroz (2014), afirman que los migrantes, reciben un trato 
desigual ante el acceso de bienes y servicios que brinda el Estado, además, la falta de sanciones de quienes ejercen o niegan este servicio propicia que continúen actos violatorios en contra de los migrantes extranjeros.

Por otro lado, el factor de la sub-escala de vulnerabilidad cultural conservó 21 reactivos iniciales y se excluyeron $7(14,15,17,19,20,21,40)$, lo cuales no cumplieron con el peso factorial necesario durante el AFE. Esto se debe a que la escala hace referencia a elementos culturales despectivos, y los eliminados iban en sentido afectivo, por lo tanto, los reactivos tenían una postura opuesta. Este factor hace inminente las actitudes que tiene la población mexicana frente a los migrantes extranjeros a través de las muestras de estereotipo y prejuicio (Pizarro, 2008). Los procesos de naturalización de estos elementos culturales (xenofobia, estereotipo y prejuicio) hacia los migrantes se manifiestan en la incomprensión del sufrimiento de este grupo social y carecen de empatía ante la población vulnerable (Holmes, 2016). Por tanto, cuando el sujeto se encuentre en una posición de contacto con los migrante, este puede ejercer actos que atenten con el bienestar físico y psicológico del migrante, atenuándole la carencia de sanciones que el Estado interpone.

Los resultados del análisis demostraron que las sub-escalas de vulnerabilidad estructural y vulnerabilidad cultural tienen una relación palpable, conforme lo señalaron Bustamante (2010) y Quiroz (2014). De acuerdo con el grado de asociación entre los factores $(r=0,28)$, ambas subescalas tienen la misma dirección logrando una asociación mediana con un nivel de significancia de $p<.01$. Ambas sub-escalas se inclinan hacia ideologías y actos perjudiciales en contra de los migrantes extranjeros. De acuerdo con esta asociación, es posible analizar que las ideologías que se gestan a través de las relaciones simbólicas entre los sujetos, ponen 
en juego los valores, las ideas, los prejuicios que se justifican a través de la diferenciación perpetua, respecto al trato desigual que reciben por parte de la sociedad. La relación entre ambas dimensiones (vulnerabilidad estructural y vulnerabilidad cultural) se pone de manifiesto a través de la práctica social (los crímenes hacia los migrantes, leyes anti-inmigratorias, arrestos, diferencias raciales y violación de los derechos humanos), y la existencia de impunidad hacia tales hechos (Bustamante, 2010; Quiroz, 2014).

En el estudio se puso a prueba un modelo anidado, se compararon cuatro modelos nulo, 2 factores relacionados, 1 factor de segundo orden ( $\sin$ parcelas) y 1 factor de segundo orden (con parcelas). El modelo nulo carece de índices de bondad de ajuste debido a la presunción de factores, mientras que los últimos tres modelos presentaron bondad de ajuste, practica, estadística y poblacional. El modelo con los índices más altos fue el factor de segundo orden con parcelas ya que tiene mayor comprensión de relación entre los constructos (Little et al., 2002).

Adicionalmente, los modelos probados en este estudio dan cuenta de validez convergente y divergente de constructo a través de la técnica de Análisis Factorial Confirmatorio. En el primer modelo, la covarianza entre las subescalas de vulnerabilidad estructural y vulnerabilidad cultural resultó menor a los pesos factoriales lo que da cuenta de validez discriminante, mientras que los pesos factoriales resultaron mayores a .60 lo que evidencia validez convergente de constructo. El segundo modelo a través de sus indicadores manifiestos (parcelas) también dan cuenta de validez convergente de constructo, lo que implica que los reactivos contribuyen de forma pareja a cada factor, ya que convergen alta y significativamente, a su respectiva dimensión (Corral, Frías y González, 2001). 
En cuanto a las diferencias del ingreso mensual familiar en contraste con la vulnerabilidad estructural y cultural, Herranz (2008) señala que personas con un estatus socioeconómico familiar bajo son más propensas a tener actitudes xenofóbicas. Lo que podría estar relacionado con que las personas con un ingreso medio y alto tienen un nivel educativo mayor, por lo que indagan las condiciones reales de los migrantes a través de varias fuentes de información para establecer un criterio objetivo y no sólo a partir de los discursos antinmigrantes (Armendares, 2018).

Este estudio no está exento de limitaciones. Se evaluó la vulnerabilidad hacia los migrantes a través de un autoinforme que está sujeto a cierto grado de subjetividad y deseabilidad social por parte de las personas evaluadas. Es importante que en futuros estudios se utilicen otros métodos alternativos, por ejemplo, estudios longitudinales que permitan evaluar el ajuste y estabilidad de los modelos presentados en este trabajo. Por otro lado, la escala fue aplicada a inicios de la declaración oficial de la pandemia COVID-19, por lo tanto, se desconoce si este evento tuvo algún efecto sobre la percepción de la población ante los migrantes extranjeros.

A pesar de las limitaciones antes mencionadas, este trabajo cuenta con implicaciones importantes que podrían ser útiles para futuros trabajos de investigación, desarrollo de políticas públicas enfocadas hacia la población migrante y para aquellos que mantienen contacto directo con este colectivo vulnerable. Este estudio se centró en analizar la vulnerabilidad hacia los migrantes en población del noroeste de México, no obstante, es importante que futuras investigaciones estudien dicho constructo en otros contextos culturales para verificar las diferencias existentes que se puedan derivar de la cultura, respecto a la vulnerabilidad hacia la población migrante como sujetos de derecho. Adicionalmente se recomienda que esta escala 
sea aplicada en poblaciones particulares, por ejemplo, académicos, legisladores, servidores públicos y grupos de la sociedad civil que tengan contacto con migrantes nacionales y extranjeros.

El presente trabajo será de suma utilidad para los interesados en evaluar la vulnerabilidad hacia el migrante a través de investigaciones con enfoques sociológicos, jurídicos y psicológicos, que puedan contribuir desde su campo de acción al desarrollo de programas preventivos o de sensibilización orientados a la sociedad en general y a los organismos públicos, con el propósito de que reconozcan los derechos humanos de los migrantes, evitar escenarios de victimización arbitraria y revictimización, a fin de erradicar las manifestaciones de prejuicio, racismo y discriminación hacia este segmento de la población en México.

\section{REFERENCIAS}

Aday, L. (1994). Health Status of Vulnerable Populations. Annual Review of Public Health, 15(1), 487-509. https://doi.org/10.1146/annurev. pu.15.050194.002415

Adger, W. N. (2006). Vulnerability. Global Environmental Change, 16, 268281. https://doi.org/10.1016/j.gloenvcha.2006.02.006

Armendares, P.E. (2018). La política migratoria de Trump: Impacto para los migrantes mexicanos y sus comunidades. México: Instituto Belisario Domínguez.

Bentler, P. (2007). On tests and indices for evaluating structural models. Pers. Individ. Differ. 42, 825-829. https://doi.org/10.1037/00332909.88.3.588

Bentler, P., y Bonet, D. (1980). Significance Tests and Goodness of Fit in the Analysis of Covariance Structure. Psychological Bulletin, 88(3), 588606. https://doi.org/10.1037/0033-2909.88.3.588 
Brooks, N. (2003). Vulnerability, risk and adaptation: A conceptual framework. Inglaterra: Tyndall Centre for Climate Change Research.

Bustamante, J. (2003). La construccion social de la vulnerabilidad de los migrantes. En D. Godenau, V. Zapata, J. Fariña, y B. Hernández (Eds.), La inmigración irregular. Aproximación multidisciplinar (pp. 19-45). Área de Desarrollo Económico.

Bustamante, J. (2010). Los grandes problemas de México. En B. Torres y G. Vega (Eds.), 1: Vol. XII (pp. 205-204). El Colegio de México.

Casillas, R. (2011). Los migrantes indocumentados: su vulnerabilidad y la nuestra. En N. Armijo (Ed.), Migración y seguridad: el nuevo desafio en México (pp. 145-164). Colectivo de Análisis de la Seguridad con Democracia, A.C.

Corral, V., Frías, M., y González, D. (2001). Análisis cuantitativos de variables latentes. Hermosillo: UNISON.

Cutter, S. L. (1996). Vulnerability to environmental hazards. Progress in Human Geography, 20(4), 529-539. https://doi. org/10.1177/030913259602000407

Field, A. (2013). Discovering Stadistics Using IBM SPSS Stadistics an Sex and Drugs and Rock 'N'Roll. Los Ángeles: SAGE.

Forsyth, B. W., Horwitz, S. M., Leventhal, J. M., Bruger, J., y Leaf, P. J. (1996). The child vulnerability scale: an instrument to measure parental perceptions of child vulnerability. Journal of pediatric psychology, 21(1), 89-101. https://doi.org/10.1093/jpepsy/21.1.89

González, E. (2010). La vulnerabilidad de los grupos migrantes en México. En L. Meza y M. Cuéllar (Eds.), La vulnerabilidad de los grupos migrantes en México. México: Universidad Iberoamericana.

González, J. (2009). Manual básico de SPSS. Manual de introducción a SPSS. Chile: Universidad de Talca.

González-Fagoaga, J. E. (2010). La vulnerabilidad de los grupos migrantes en México. Migraciones internacionales, 5(3), 233-239. 
Hernández, R. Fernández, C. y Baptista, L. (2014). Metodología de la Investigación. México: McGraw Hill.

Hernández-Rosete, D., Sanchez, G., Pelcastre, B., y Juárez, C. (2005). Del riesgo a la vulnerabilidad. Bases metoológica para comprender la relación entre violencia sexual e infección por VIH/ITS en migrantes clandestinos. Salud Mental, 28(5), 20-26.

Herrnaz, G. (2008). Xenofobia: Un estudio comparativo en barrios y municipios almerienses. Revista Espanola de Investigaciones Sociologicas, 121(1), 107-132. https://doi.org/10.2307/40184847

Holmes, S. M. (2016). Fruta fresca, cuerpos marchitos: trabajadores agrícolas migrantes en Estados Unidos. Quito: Abya Yala.

Hu, L., y Bentler, P. (1999). Cutoff criteria for fit indexes in covariance structure analysis: Conventional criteria versus new alternatives. Structural Equation Modeling, 6(1), 1-55. https://doi. org/10.1080/10705519909540118

Kasperson, R. E., y Dow, K. (2005). Vulnerable people and places. En R. Norgaard y D. Rapport (Eds.), Ecosystems and Human Well-being: Current State and Trends (pp. 145-162). Millennium Ecosystem Assessment.

Little, Todd, Cunningham, William, Shahar, Golan y Widaman, Keith. (2002). To parcel or not to parcel: Exploring the question, weighing the merits. Structural Equation Modeling, 9(2), 151-173. https://doi. org/10.1207/S15328007SEM0902_1

Nogueira, M. J., Barros, L., y Sequeira, C. (2017). Psychometric properties of the psychological vulnerability scale in higher education students. Journal of the American Psychiatric Nurses Association, 23(3), 215222. https://doi.org/10.1177/1078390317695261

Organización de las Naciones Unidas, (2003). Informe sobre la situación social en el mundo. Naciones Unidas.

París, M. D. (2012). Vulnerabilidad de jóvenes migrantes en el cruce indocumentado de la frontera México-Estados Unidos. Trace, 62, 2135. https://doi.org/10.22134/trace.62.2012.455 
Pineda, J. R. (2020). Vulnerability to HIV/AIDS in gay and bisexual individuals during migration: the case of Colombian immigrants residing in Spain. Saúde e Sociedade, 29, e190298. https://doi. org/10.1590/S0104-12902020190298

Pinsker, D. M., McFarland, K., y Stone, V. E. (2011). The social vulnerability scale for older adults: An exploratory and confirmatory factor analytic study. Journal of Elder Abuse \& Neglect, 23(3), 246-272. https://doi. org/10.1080/08946566.2011.584049

Pituch, K. A., y Stevens, J. P. (2016). Applied multivariate statistics for the social sciences: Analyses with SAS and IBM's SPSS. New York: Routledge. https://doi.org/10.4324/9781315814919

Pizarro, C. (2008). La vulnerabilidad de los inmigrantes bolivianos como sujetos de derechos humanos: experimentando la exclusión y la discriminación en la región metropolitana de la ciudad de Córdoba Índice. Comunicación presentada en Concurso de Proyectos de Investigación sobre Discriminación.

Quiroz,Y.S.(2013). Vulnerabilidad: Un concepto para pensar las migraciones inernacionales. En M. E. Anguiano y R. Cruz (Eds.), Migraciones internacionales, crisis y vulnerabilidades: perspectivas comparadas. México: El Colegio de la Frontera Norte.

Quiroz, Y. S. (2014). Transmigración de centroamericanos por México: su vulnerabilidad y sus derechos humanos. México: El Colegio de La Fontera Norte.

Restrepo, L., y González, J. (2007). De Pearson a Spearman. Revista Colombiana de Ciencias Pecuarias, 20(2), 183-192.

Salgado, N., González, T., Bojorquez, I., y Infante, C. (2007). Vulnerabilidad social, salud y migración México-Estados Unidos. Salud Pública En México, 49. https://doi.org/10.1590/S0036-36342007001000003

Schumacker, R. E., y Lomax, R. G. (2004). A beginner's guide to structural equation modeling. London: Psychology press.

Seward, R. J., Bayliss, D. M., y Ohan, J. L. (2018). The Children's Social Vulnerability Questionnaire (CSVQ): Validation, relationship with 
psychosocial functioning, and age-related differences. International Journal of Clinical and Health Psychology, 18(2), 179-188. https:// doi.org/10.1016/j.ijchp.2018.02.001

Sinclair, V. G., y Wallston, K. A. (1999). The development and validation of the Psychological Vulnerability Scale. Cognitive Therapy and Research, 23(2), 119-129. https://doi.org/10.1023/A:1018770926615

Sofronoff, K., Dark, E., y Stone, V. (2011). Social vulnerability and bullying in children with Asperger syndrome. Autism, 15(3), 355-372. https:// doi.org/10.1177/1362361310365070

Envío a dictamen: 8 de junio de 2021

Reenvío: 6 de julio de 2021 Aprobación: 10 de agosto de 2021

Fernanda Guadalupe Rascón Arriaga. Licenciada en Psicología, maestra en Ciencias Sociales, estudiante de doctorado en Ciencias Sociales del Posgrado Integral de Ciencias Sociales, Universidad de Sonora. Líneas de investigación: Problemas psicosociales, transnacionalismo y migración. Correo electrónico: fergpe_93@hotmail.com

Nissa Yaing Torres Soto. Licenciada en Piscología Social Comunitaria, maestra en Innovación Educativa, estudiante de doctorado en Ciencias Sociales del Posgrado Integral de Ciencias Sociales, Universidad de Sonora. Líneas de investigación: problemas psicosociales, calidad de vida y bienestar. Correo electrónico: yaing.torres@gmail.com

Héctor Francisco Vega Deloya. Licenciado en Sociología, maestro en Estudios Hispánicos y doctor en Antropología, Profesor-investigador de tiempo completo del Departamento de Historia y Antropología de la Universidad de Sonora. Líneas de investigación: transnacionalismo y migración, etno-política, medio ambiente y derechos ambientales y estudios culturales. Correo electrónico: hector.vega@unison.mx 